\title{
Local time dependence of the equatorial counter electrojet effect in a narrow longitudinal belt
}

\author{
S. Alex and S. Mukherjee \\ Indian Institute of Geomagnetism, Dr. Nanabhai Moos Marg, Colaba, Mumbai - 400 005, India \\ (Received February 14, 2001; Revised August 2, 2001; Accepted September 8, 2001)
}

\begin{abstract}
Irregular occurrence characteristics of the equatorial counter electrojet phenomena at different longitudinal zones are investigated using the magnetic data of the horizontal component ' $H$ ' at the two equatorial stations Trivandrum and Addis Ababa, separated in longitude by about $40^{\circ}$. The limited longitudinal extent of the counter electrojet (CEJ) events are examined on the basis of the occurrence of the phenomenon at different local time intervals. Importance of the solar semi diurnal tides in modifying the equatorial counter electrojet phenomena at Trivandrum and Addis Ababa is discussed during these local time groups. Dominance of semi-diurnal tidal modes of varying magnitude is found to influence the local time occurrence behaviour of CEJ events over the equator. Peculiarities of both the change in amplitude and phase pattern of the diurnal variation of the ' $H$ ' component within the narrow longitude sector, especially during the noon hours, indicate the modulation of the diurnal variation of the equatorial electrojet currents by vertical wind shears of highly localised nature.
\end{abstract}

\section{Introduction}

Abnormally large range of diurnal variation of the horizontal component of the geomagnetic field was found at the equatorial observatory Huancayo by Bartels and Johnston (1940), Egedal, (1947) and Chapman (1951). Egedal (1948) attributed this feature to be due to a narrow band of enhanced eastward current centered on the magnetic equator. Chapman (1951) termed this phenomenon as "equatorial electrojet." Subsequently Gouin (1962) noticed the interesting aspect of the reversal in direction of the normal northward directed field of the horizontal component around midday hours at the equatorial station Addis Ababa. Gouin and Mayaud (1967) explained this negative depressions of the regular $H$-field to be the effect of a westward flow of electrojet current and named this phenomenon as "equatorial counter electrojet." Similar occurrences in the diurnal variation in the ' $H$ ' component were observed at other equatorial stations, Ibadan (Onwumechilli, 1963), Zaria (Hutton and Oyinloye, 1970) and Trivandrum (Sastry and Jayakar, 1972).

In continuation of the findings by Gouin (1962) about the counter electrojet (CEJ) event at Addis Ababa, Rao and Rajarao (1963) observed that the magnetograms at Trivandrum and Annamalainagar did not show any reversal for the same days 3rd and 5th January 1962 as examined by Gouin. Rastogi (1973) reported cases when the daytime depressions in the ' $H$ ' component differed very much between the equatorial station, Addis Ababa and the magnetograms at the Indian equatorial station, Kodaikanal, concluding that the phenomenon of afternoon depression in ' $H$ ' is local-

Copy right (C) The Society of Geomagnetism and Earth, Planetary and Space Sciences (SGEPSS); The Seismological Society of Japan; The Volcanological Society of Japan; The Geodetic Society of Japan; The Japanese Society for Planetary Sciences. ized in longitude and on some occasions the events may not occur on the same day even at locations separated by a narrow longitude of 2 hours. Morphological characteristics of the counter electrojet phenomena have been studied extensively by Rastogi (1974a), Mayaud (1977) and Marriott et al. (1979). Kane (1973) and Rastogi (1974a) examined the cases of various counter electrojet events at different longitudes and they pointed out that the depressions of the $H$-field at these longitudes are varying in nature and discussed the feature of limited longitudinal extent. However, the results of Onwumechili and Akasofu (1972) depicted the occurrence of the counter electrojet over a wider longitude on some occasions. Mayaud (1977) in his review, demonstrated the morphological characteristics of limited latitudinal and longitudinal extent of counter electrojet events on many days. It was illustrated that the irregularity in the occurrence phenomena would cause frequent variations of the longitude extension of a given event. The perception of Mayaud (1977) was that a given event is never restricted to a narrow longitude band and is commonly spread over 5-8 hour in longitude and sometimes occurs over a much wider band. Kane and Trivedi (1981) demonstrated the aspect of limited confinement of the longitudinal extent in a less than $30^{\circ}$ belt by comparing the ' $H$ ' variations at the two locations in the Brazilian region across the East and the West coast in the South American sector. Furthermore, study by Rangarajan and Rastogi (1993) on the significant features of counter electrojet at locations separated in a narrow longitude suggested the limited correspondence of the influence of the westward current over the equatorial latitudes separated by $2-3$ hour in longitude.

While the earlier reports considered counter electrojet in general, the present work emphasizes on the local time of occurrence of the event in reference to their diurnal features 


\section{Strong Electrojet at both TRD \& AAE}
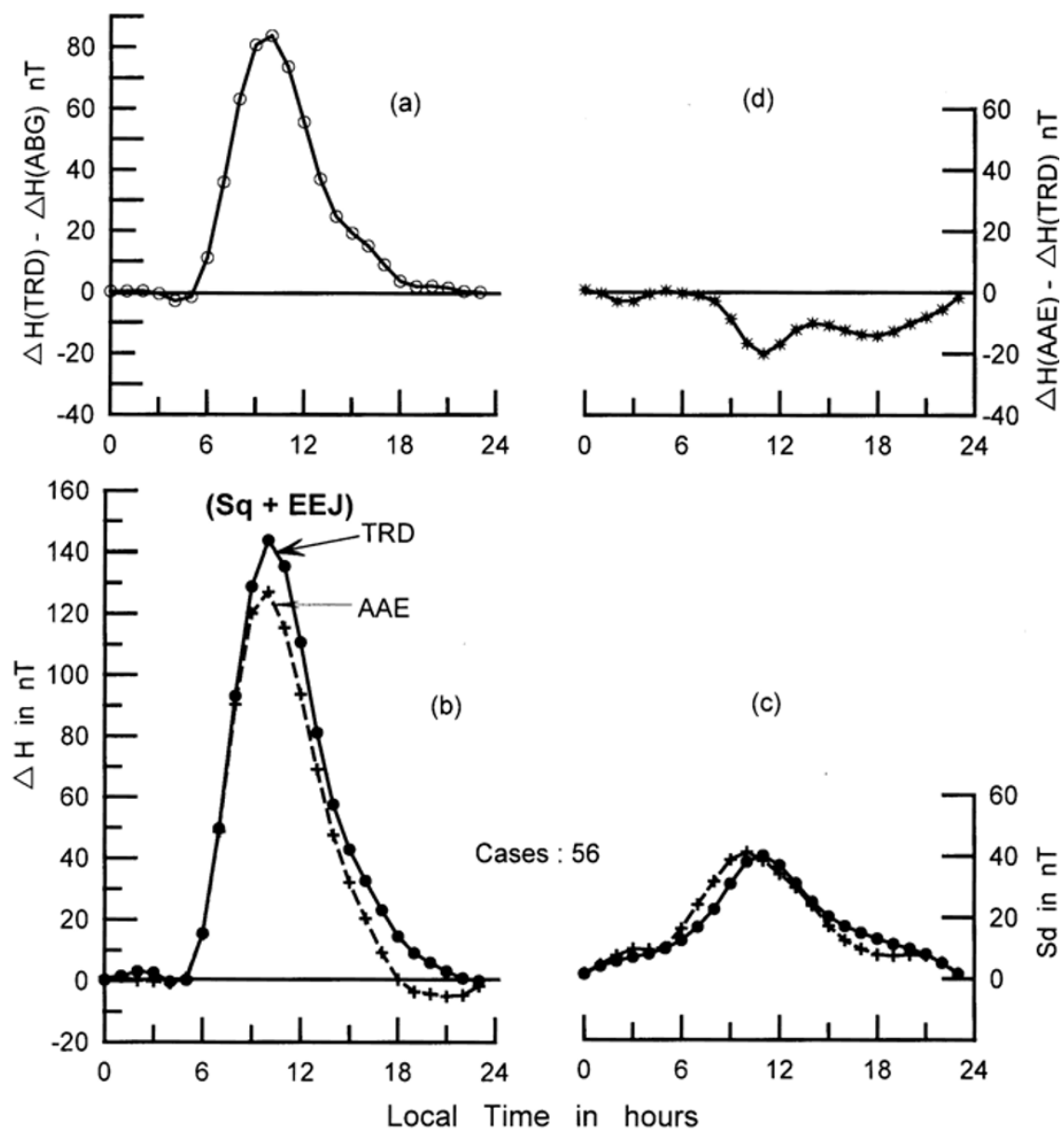

Fig. 1. (a) Equatorial electrojet (EEJ) strength averaged for 56 strong jet days in the Indian longitude are shown. (b) Average diurnal variation curves $(\Delta H)$ at Trivandrum (TRD) and Addis Ababa (AAE). Day-to-day variability (c) is represented for each hour by the Sd curves. Longitudinal difference field (d) $(\Delta H(\mathrm{AAE})-\Delta H(\mathrm{TRD}))$ depicts the hourly departures.

at equatorial stations situated in a narrow longitude belt. The study also focuses on the magnitude of day-to-day variability in the equatorial electrojet (EEJ) and counter electrojet (CEJ) events at the two equatorial stations, separated in a narrow longitude by about $40^{\circ}$. The irregular occurrence of the counter electrojet events with longitude has led to the present exercise of classification of such counter electrojet events into various Local Time (LT) intervals, to ascertain and interpret the associated dynamic processes and its progressional behaviour with local time.

\section{Database}

Database of the study mainly comprise of the Horizontal component ' $H$ ' from the two equatorial stations Trivandrum (TRD) (dip lat. $0.2^{\circ} \mathrm{N}$, geog. lat. $8^{\circ} .48 \mathrm{~N}$, long. $77^{\circ} \mathrm{E}$ ) and Addis Ababa (AAE) (dip lat. $0.3^{\circ} \mathrm{N}$, geog. lat. $9^{\circ} .03 \mathrm{~N}$, long. $38^{\circ} \mathrm{E}$ ) for the period 1991-1993. The EEJ strength is computed for the Indian longitude $\left(75^{\circ} \mathrm{EMT}\right)$ by $[\Delta H(\mathrm{TRD})-$ $\Delta H(\mathrm{ABG})]$ where TRD is an equatorial station and Alibag (ABG) (dip lat. $13.2^{\circ} \mathrm{N}$, geog. lat. $18^{\circ} .61 \mathrm{~N}$, long. $73^{\circ} \mathrm{E}$ ) is a station outside the influence of the equatorial electrojet. $\Delta H$ refers to the diurnal variation of ' $H$ ' after the removal of mean midnight level. Unfortunately similar exercise was not possible for Addis Ababa due to the lack of a station outside the jet influence in the same sector.

Classification of the days to compute the mean diurnal variations ' $\Delta H$ ' and the electrojet strength $[\Delta H(\mathrm{TRD})-$ $\Delta H(\mathrm{ABG})]$ is done only for days with $A p<10$, to avoid any major disturbance effect on the data set.

\section{Methodology}

The method of Harmonic Analysis is employed on the hourly values of ' $H$ ' for the days chosen for the period 1991-1993. Thus 1440 synthesized values are obtained corresponding to each one minute value for each day under consideration, where the deduced $H_{i}$ is expressed as.

$$
\begin{aligned}
H_{i} & =\sum_{j=1}^{6} \operatorname{amp}(j) \times \sin \left[(\omega \times i \times j)+\phi_{j}\right] \quad \text { where, } \\
\omega & =(2 \pi / L), \quad L=1440 \\
i & =0,1,2,3, \ldots, 1439 \text { minute values } \\
j & =1,2,3, \ldots, 6 \text { harmonics } \\
\phi_{j} & =\text { phase of } j \text { th harmonics. }
\end{aligned}
$$

This method is employed to re-compute the hourly values 
Occurrence of MCEJ (0600 LT)
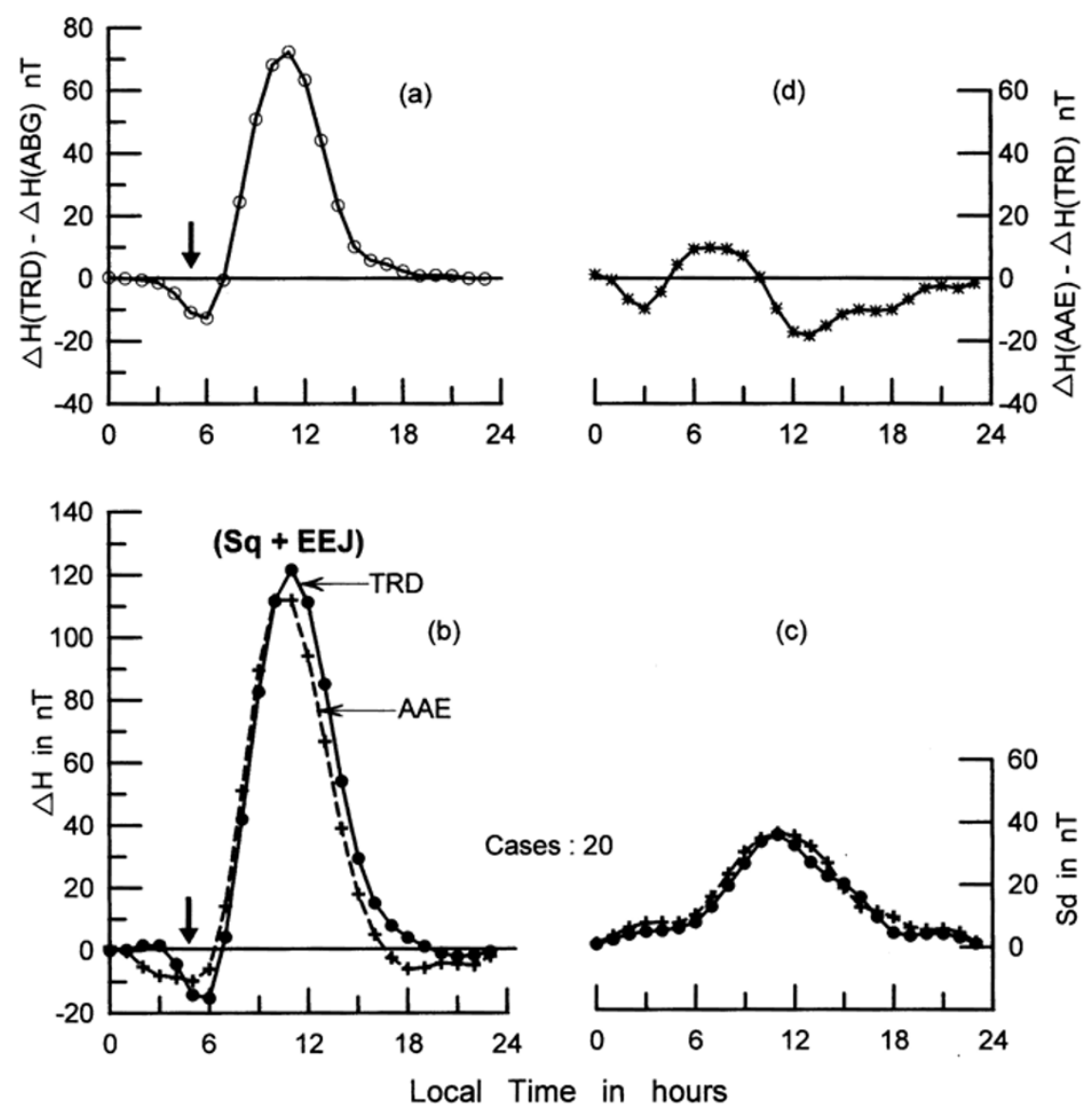

Fig. 2. Mean diurnal curves (b) at Trivandrum (TRD) and Addis Ababa (AAE) for 20 days of MCEJ (0600 LT). The arrow marked on the average curve of electrojet strength (a) against the time of MCEJ. Sd curves (c) represent the day-to-day variability pattern. The difference field (d) shows the hourly departures for the MCEJ days.

for each minute to assess the precise orientation of LT based on their geographic longitudes at both the locations TRD and AAE.

The selection criteria to define the electrojet strength is done using the parameter $[\Delta H(\mathrm{TRD})-\Delta H(\mathrm{ABG})]$ for Strong Electrojet (SEJ) and CEJ categories of days (Table 1). $\Delta H$ from the equatorial station, AAE, is used to compare the effects of $(\mathrm{Sq}+\mathrm{EEJ})$ on SEJ and CEJ events observed at this station with that of TRD at $77^{\circ} \mathrm{E}$. As a measure of the variability, the Standard deviation (Sd) for each hour is computed for the various data sets.

\section{Observations and Results}

\subsection{SEJ}

SEJ days selected from the electrojet index $[\Delta H(\mathrm{TRD})-$ $\Delta H(\mathrm{ABG})]$, are in the category with the magnitude of noon jet strength $\geq 70 \mathrm{nT}$, with the absence of any strong CEJ occurrence on these days. Curve shown in the upper left block (Fig. 1(a)) represents the average electrojet strength variation for the 56 days chosen. Mean diurnal variation curves (Fig. 1(b)) $\Delta H$ (TRD) and $\Delta H$ (AAE) depict the average $(\mathrm{Sq}+\mathrm{EEJ})$ contribution at the two locations. The expected quiet day variation is evident at both the stations, showing an increase in the field with sunrise reaching maximum near noon and taking over to the night level by evening. Considering the pattern of variations at TRD and AAE, mean diurnal variations at both the stations match quite well in the morning hours, indicating the correspondence. However, the afternoon field at AAE which comes under the electrojet influence drops much faster. As the number of cases examined are distributed over various days, the magnitude of day-to-day variability is expressed by the Sd computed for each hour representing the variability index for respective locations. Diurnal variation curves of $\mathrm{Sd}$ at TRD and AAE are shown in the lower right block (Fig. 1(c)). The diurnal trend in the variability is seen to follow the mean diurnal variation trend in $\Delta H$ with negligibly low departures in the magnitude of variability between the two stations. The mean hourly departures in the diurnal magnitude of the ' $H$ ' field at AAE with respect to that at the station TRD are represented by the difference field ( $\Delta H(\mathrm{AAE})-\Delta H(\mathrm{TRD}))$ as shown in the Fig. 1(d). The negative excursion in the afternoon hours is indicative of the average magnitude of the deficit field at AAE. We next consider the difference in the diurnal pattern when CEJ events occur at different local times. 
Individual cases of MCEJ ( 0600 LT )

$$
\text { ' } \triangle H^{\prime}
$$

(a ) at both TRD \& AAE $\quad$ (b) at TRD only
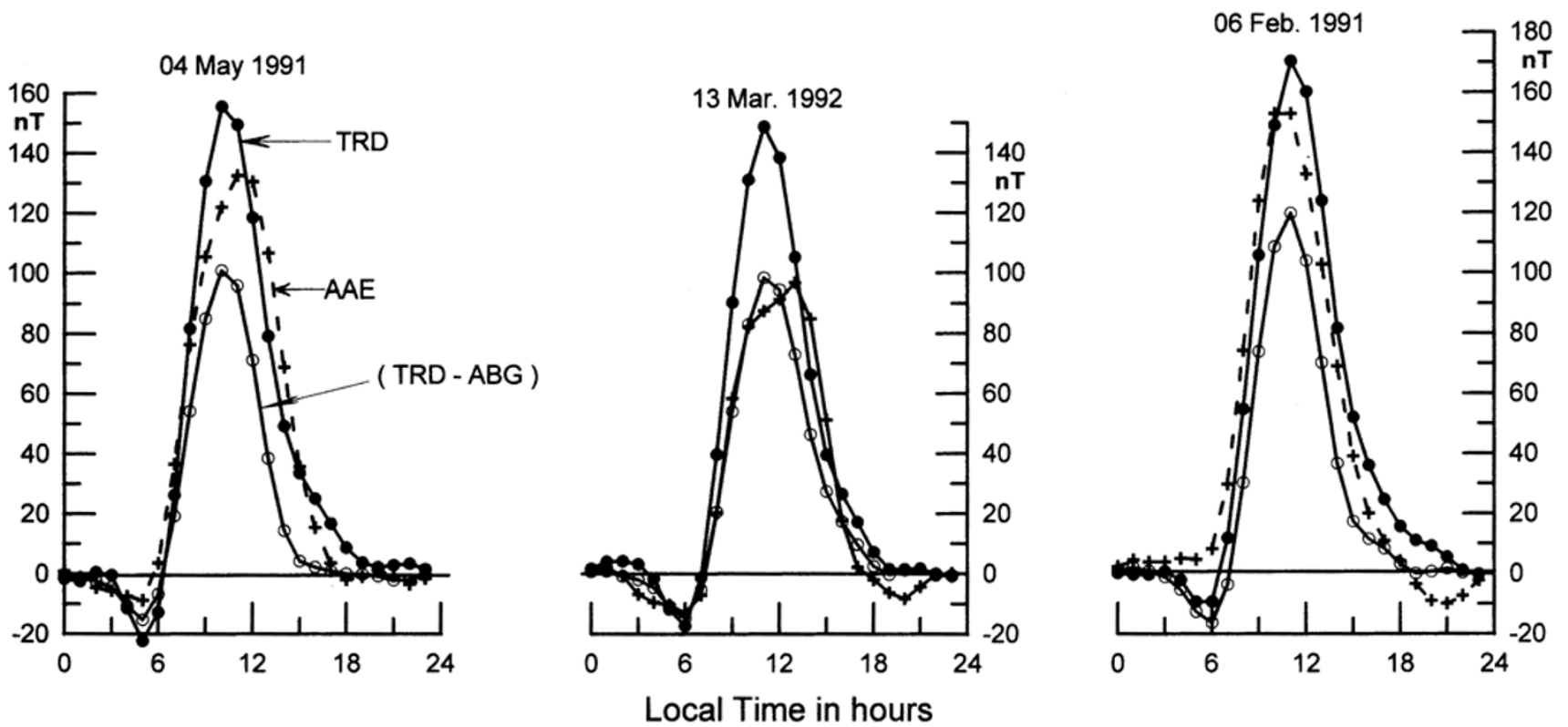

Fig. 3. Diurnal variation curves (a) at TRD and AAE, on 4 May 1991 and 13 March 1992, show simultaneous occurrence of MCEJ around 0600 LT. 6 Feb. 1991 (b) is an example with the MCEJ present at TRD only. $\Delta H$ (TRD - ABG) curves are shown for reference.

Individual cases of MCEJ ( 0700 - 0800 LT)

$$
' \Delta H^{\prime}
$$
(a) at both TRD \& AAE
(b ) at TRD only
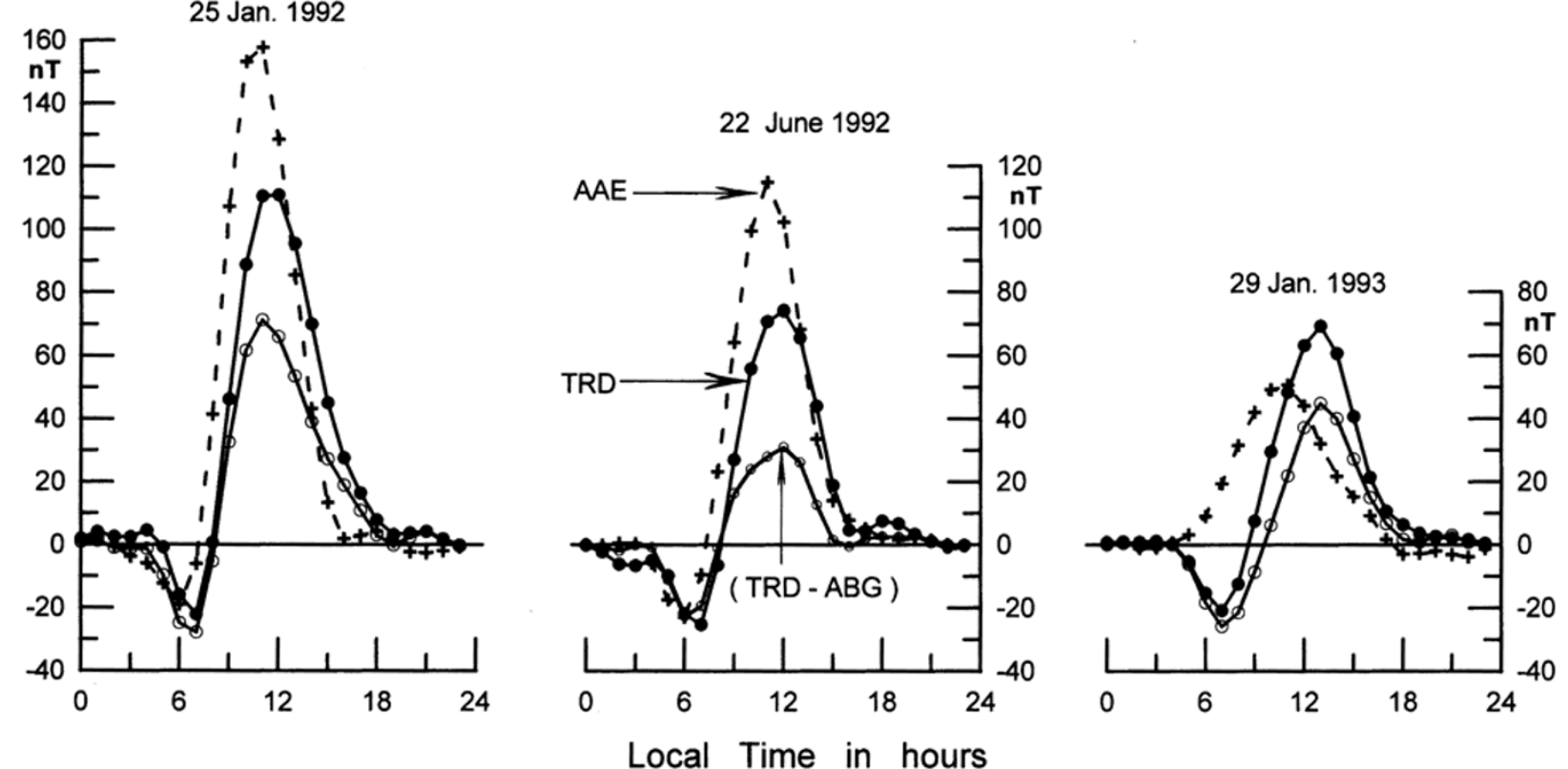

Local Time in hours

Fig. 4. Diurnal variation curves (a) at TRD and AAE, on 25 Jan. 1992 and 22 June 1992, show simultaneous occurrence of MCEJ (0700-0800 LT). 29 Jan. 1993 (b) is a sample case of strong MCEJ (0700-0800 LT) present at TRD only. 


\section{Occurrence of NCEJ ( $1100-1200 \mathrm{LT})$}
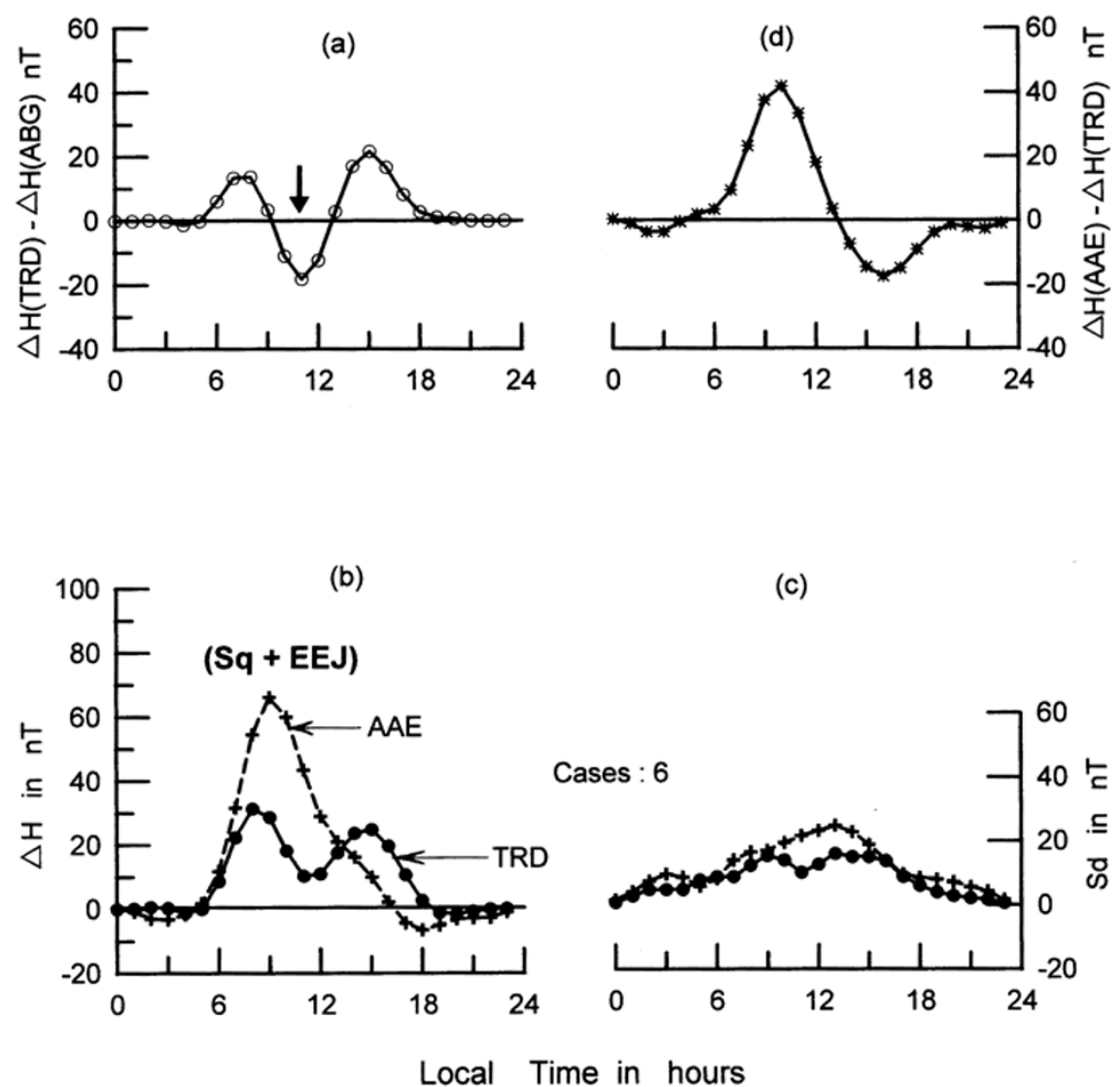

Fig. 5. The occurrence time of NCEJ is shown by the arrow in (a). The mean diurnal variation curves (b) at Trivandrum (TRD) and Addis Ababa (AAE) for 6 cases of NCEJ (1100-1200 LT). Departures in the day-to-day variability (c) has a prominent noon magnitude for AAE. Semi-diurnal pattern is evident in the difference curve $(\mathrm{d})$.

\subsection{Occurrence of morning counter electrojet (MCEJ) at $0600 \mathrm{LT}$}

The morning counter electrojet (MCEJ) events were chosen based on the time of occurrence of prominent negative field in $\Delta H$ around $0600 \mathrm{LT}$ at TRD as well as from the parameter $[\Delta H(\mathrm{TRD})-\Delta H(\mathrm{ABG})]$. Average curve of electrojet strength given in Fig. 2(a) clearly shows the morning depression indicative of the time period of CEJ averaged over the 20 days chosen for the study. Diurnal variation curves of $\Delta H(\mathrm{TRD})$ and $\Delta H(\mathrm{AAE})$, shown in Fig. 2(b), indicate the presence of MCEJ at both the locations. CEJ occurrence in the morning hours does not seem to alter the trend of diurnal variation at any of the stations, thus strictly following the SEJ characteristics of almost a one to one correspondence in the pre-noon hours for TRD and AAE. The rate of decrease in the field at AAE in the afternoon hours is rather sharp; thus showing a consistent departure during this period till late evening hours. Here again, the collapse in the evening field at AAE is dominant. Sd curves, shown in Fig. 2(c), systematically follow the trend of diurnal variation in $\Delta H$, but no significant difference is observed in the hour to hour variability pattern between the two stations as is the case with SEJ. The difference curve (Fig. 2(d)) exhibits a positive peak in the pre-noon hours and a clear depression in the afternoon hours.

Illustrative diagrams to explain the individual day occurrence features at TRD and AAE are shown in Fig. 3. The two days 04 May 1991 and 13 March 1992 are two typical cases, showing a distinct minimum in the ' $H$ ' field observed around 0600 hours at both the stations. The day 06 February 1991, is a sample case to depict the absence of the morning depression at AAE. In the event of occurrence of MCEJ at $0600 \mathrm{LT}$ at both the locations or at TRD alone, the noon ' $H$ ' field at TRD shows a higher magnitude than that at AAE. In order to examine MCEJ in a more broader time scale, cases are chosen for the days when the diurnal variation curves of $\Delta H(\mathrm{TRD})$ and $\Delta H(\mathrm{AAE})$ showed clear negative depression during 0700-0800 LT. However the occurrences of MCEJ during this interval are found to be few in number (six cases only) and hence the representative cases have been chosen and are presented in Fig. 4. Of the six cases, the common occurrence could be found in four cases. In contrast to the reduced noon magnitude for AAE (Fig. 3), the magnitudes of the northward field as shown by the diurnal variation curves at AAE on 25 January 1992 and 22 June 1992 are considerably enhanced than that at TRD. The day 29 January 1993, is a sample case which shows the absence of CEJ at AAE while the diurnal variation at TRD 


\section{Occurrence of ACEJ (1300 - 1400 LT)}
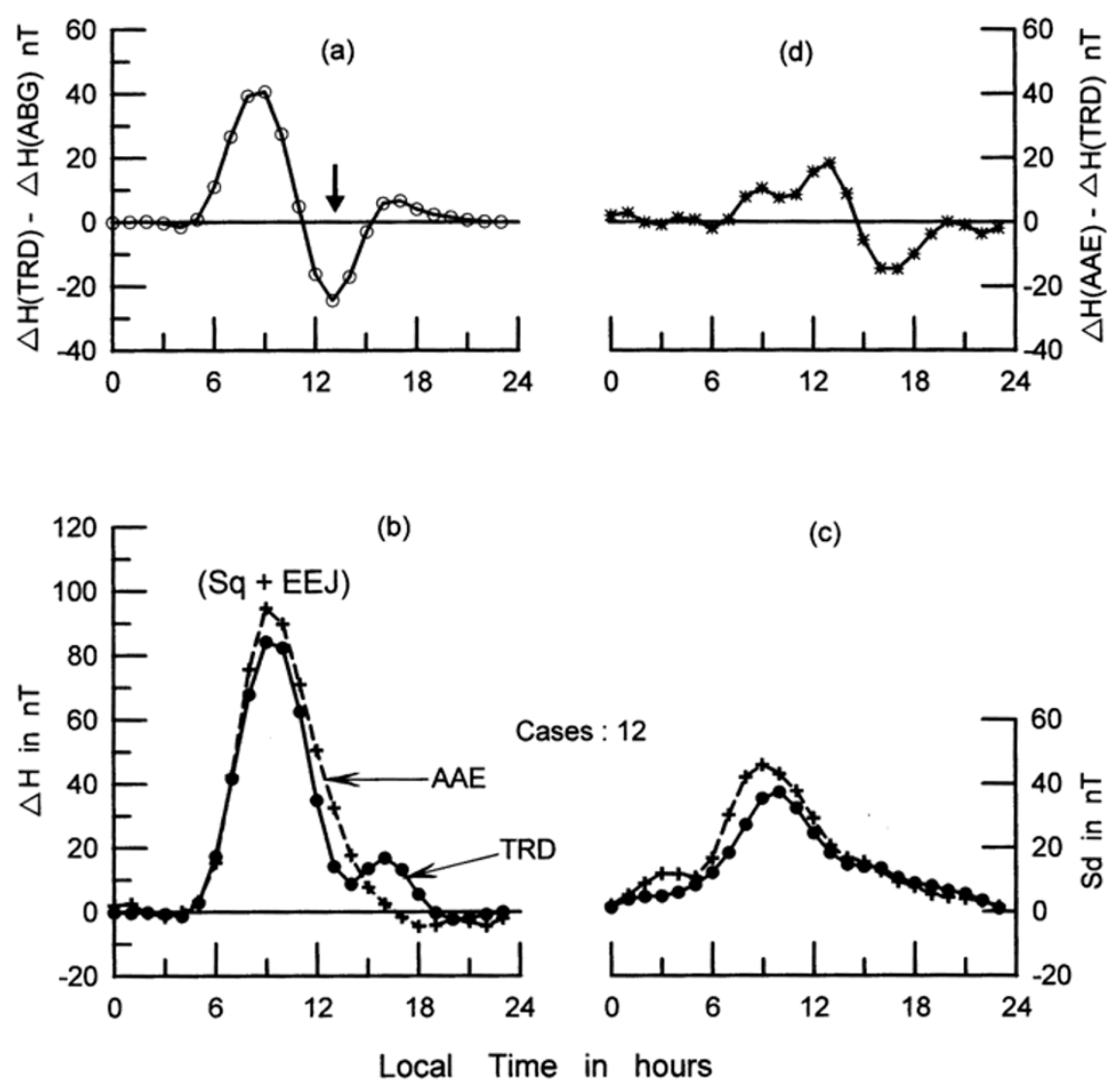

Fig. 6. The occurrence time of ACEJ is shown by the arrow in (a). The mean diurnal variation curves (b) at Trivandrum (TRD) and Addis Ababa (AAE) for 12 cases of ACEJ (1300-1400 LT). Departures in the day-to-day variability (c) follows the trend of diurnal field. Semi-diurnal trend persists with reduced magnitude as shown by the difference curve $(d)$.

shows considerable negative depression in the $H$ field during 0700-0800 LT hours. From Fig. 4, it is evident that on days of strong MCEJ occurring at 0700-0800 LT, simultaneously at both the locations, the noon field is much more enhanced at AAE than that at TRD, unlike the case of MCEJ occurring at $0600 \mathrm{LT}$. This phenomenon of the reduction in magnitude at TRD on these two typical cases, very well bring out the dominance of a westward field of the diurnal component as a whole at the location. However, in the event of absence of MCEJ at AAE, the noon field is subdued at AAE, while TRD shows a greater magnitude of the northward field component. Abnormalities of this nature are to be investigated in detail to explain the variable day-to-day dynamics.

In view of examining the varied influence of the electrojet current system over these two longitudinal sectors, cases of CEJ events are chosen depending on their appearance during the pre-noon hours or post-noon hours over the Indian longitude.

\subsection{Noon counter electrojet (NCEJ) at 1100-1200 LT}

The occurrence of the noon time CEJ is a rare phenomena. Hence the number of events chosen under such category are six only. Out of these six cases, five days happened to be in December solstice and one day in August.
Days are chosen in such a way that the hourly values were present for both the stations. Examining the diurnal signatures at TRD and AAE (Fig. 5(b)), corresponding to the period of noon counter electrojet (NCEJ) marked in the curve of $[\Delta H(\mathrm{TRD})-\Delta H(\mathrm{ABG})]$ (Fig. 5(a)), the noon depression in $\Delta H(\mathrm{TRD})$ is obvious, whereas $\Delta H(\mathrm{AAE})$ shows pronounced magnitude of the northward field during noon hours, except the subdued field after 1400 LT (Fig. 5(b)). Despite the absence of any influence of westward current effecting the magnitude at AAE during the noon hours, the occurrence of the noon peak at AAE is seen well before the expected noon period. This peak in $\Delta H(\mathrm{AAE})$ at $0900 \mathrm{LT}$, coincides with the pre-noon peak of TRD. Moreover, unlike the TRD curve pattern, AAE exhibits a sharp reduction in the afternoon field. Noontime enhancement at AAE invokes to suggest the presence of a strong eastward directed current active at this location. Sd variation (Fig. 5(c)) follows the mean field variation pattern at both the locations with an enhanced noon variability at AAE. The semi-diurnal trend with a pronounced peak in the pre-noon hours and the large negative magnitude of the difference parameter $(\Delta H(\mathrm{AAE})-\Delta H(\mathrm{TRD}))($ Fig. $5(\mathrm{~d}))$ in the evening hours illustrate the varied local time influence of the electrojet current system over the two locations. 

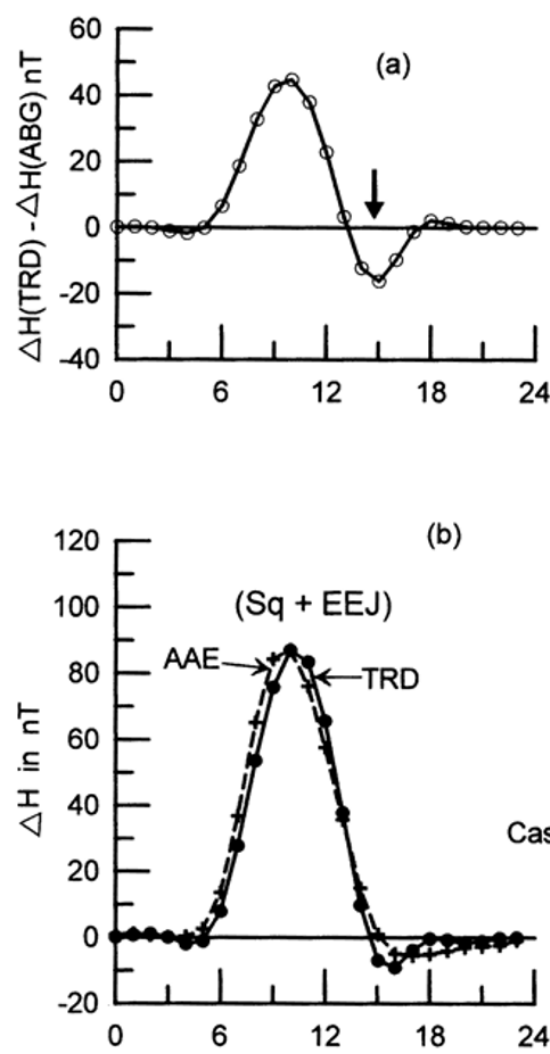

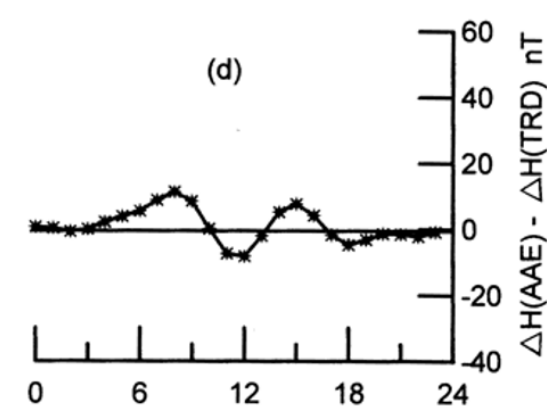

(c)

Fig. 7. The occurrence time of ECEJ is shown by the arrow (a). The mean diurnal variation curves (b) at Trivandrum (TRD) and Addis Ababa (AAE) for 24 cases of ECEJ (1500-1800 LT). No distinct departures in the day-to-day variability (c) between the two stations. Diminished difference field (d) of semi-diurnal nature still present.

\subsection{Afternoon counter electrojet (ACEJ) at 1300- 1400 LT}

Well defined asymmetry in the pre-noon and post-noon variation is evident from the average curve of the electrojet depicted by $[\Delta H(\mathrm{TRD})-\Delta H(\mathrm{ABG})]$, for the 12 cases when the counter electrojet occurred around 1300-1400 LT (Fig. 6(a)). The diurnal curve $\Delta H(\mathrm{TRD})$ shows a clear afternoon dip, indicating the CEJ effect, whereas $\Delta H$ (AAE) during the afternoon hours do not exhibit any corresponding dip (Fig. 6(b)). Subsequently, the ' $H$ ' field at AAE during the late evening hours is slightly depleted, in contrast to the peaked variation of $\Delta H(\mathrm{TRD})$. Here again the Sd curves (Fig. 6(c)) follow the diurnal trend exhibiting a larger day-to-day variability at AAE, especially during the noon hours. Diurnal asymmetry is evident from the difference curve ( $\Delta H(\mathrm{AAE})-\Delta H(\mathrm{TRD}))$ (Fig. 6(d)), suggesting the occurrence of a distinct minimum in the ' $H$ ' field at AAE, much later than the electrojet minimum found at the longitude $77^{\circ} \mathrm{EMT}$. The progressive reduction in the magnitude of the difference field with local time is indicative of the persisting special feature of the negative field at $\mathrm{AAE}$ during late afternoon hours, whereas the enhanced field in the morning hours gets diminished as the event progresses towards late afternoon hours.

\subsection{Evening counter electrojet (ECEJ) at 1500- 1800 LT}

Extensive studies on the CEJ characteristics were dealt in for evening CEJ (ECEJ) conditions by various researchers. In the present study, the signatures of the diurnal behaviour at the two locations TRD and AAE, for conditions when the average curve $[\Delta H(\mathrm{TRD})-\Delta H(\mathrm{ABG})]$ shows the presence of ECEJ during 1500-1800 LT are presented in Fig. 7(a) (24 cases chosen). An important point to note in the diurnal variation curves (Fig. 7(b)) at AAE and TRD is the absence of any morning-evening asymmetry in the variation pattern of $\Delta H(\mathrm{TRD})$ and $\Delta H(\mathrm{AAE})$ as was the case in other CEJ events presented. Signature of the CEJ occurrence is evident at AAE, but the recovery is extended, unlike the negative variation pattern at TRD around 1500-1600 LT recovering during the late evening hours. This clearly suggests that CEJ occurrences in the late afternoon hours do not influence the magnitude of the diurnal trend at both the locations. Considering the curves of Sd (Fig. 7(c)), no marked departures are evident between the locations. The difference magnitude (Fig. 7(d)) at AAE with reference to TRD depicts the dominant field of semi-diurnal nature, though the hourly differences are less pronounced in the day-to-day variability between the two locations during the ECEJ conditions.

Few individual cases to bring out the prominent features 


\section{Individual cases of strong CEJ at TRD only ' $\triangle H^{\prime}$}

(a) 28 Jan. 1991 NCEJ $(1100$ - 1200 LT $)$

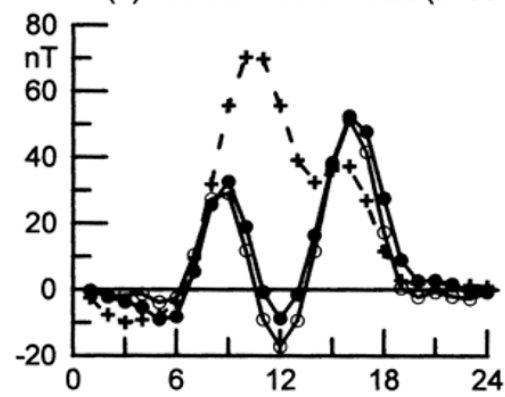

(b) 21 Jan. 1992 ACEJ (1300 - 1400 LT)
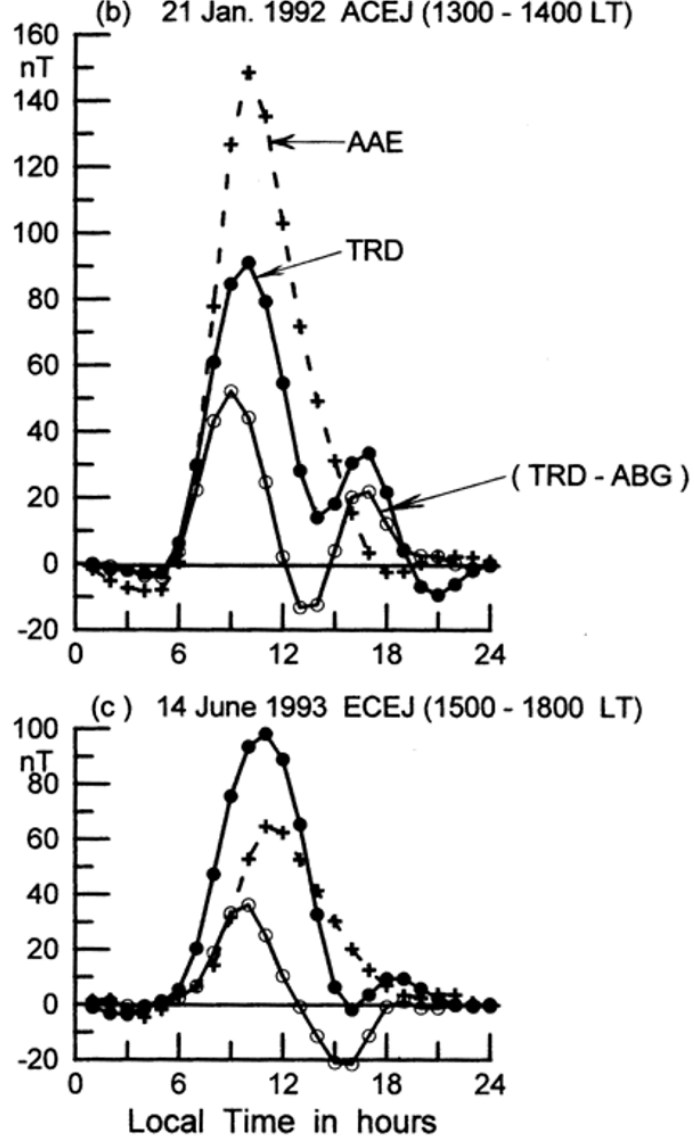

Fig. 8. Individual cases of the occurrence of strong CEJ conditions at TRD at different local times are shown. Corresponding diurnal features at AAE is also given to explain the effect of differed dynamics in each case.

of strong equatorial CEJ events during noon and afternoon hours at TRD are shown in Fig. 8. The suppression in the noon amplitude of the northward field at TRD is clearly evident on 28 January 1991, a day with a prominent NCEJ (1100-1200 LT) at TRD (Fig. 8(a)), ACEJ (1300-1400 LT) on 21 January 1992 (Fig. 8(b)) and ECEJ (1500-1800 LT) on 14 June 1993 (Fig. 8(c)), while the corresponding $\Delta H$ curves at the location AAE, do not show any trace of negative depression for the days. On the two days with strong NCEJ (Fig. 8(a)) and ACEJ (Fig. 8(b)) at TRD, the northward field in the diurnal variation curve at AAE show significantly enhanced magnitude during the noon hours com-
Individual cases of ECEJ ( 1500 - 1800 LT )

(a) prominent at $\mathrm{AAE}$.

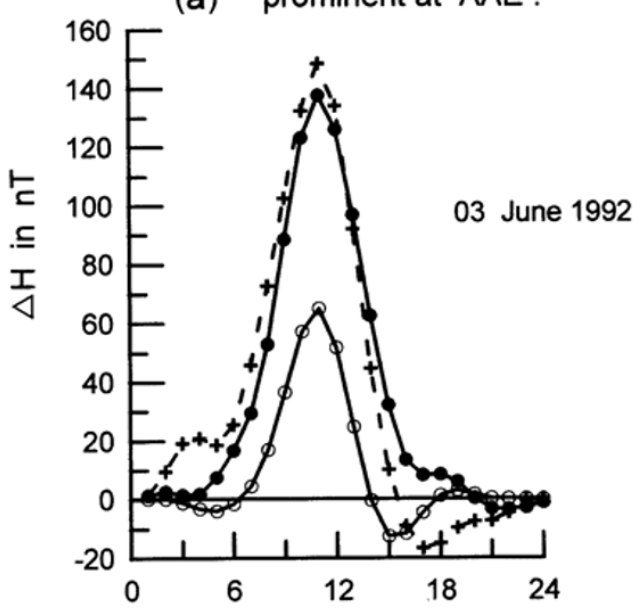

(b) prominent at TRD.

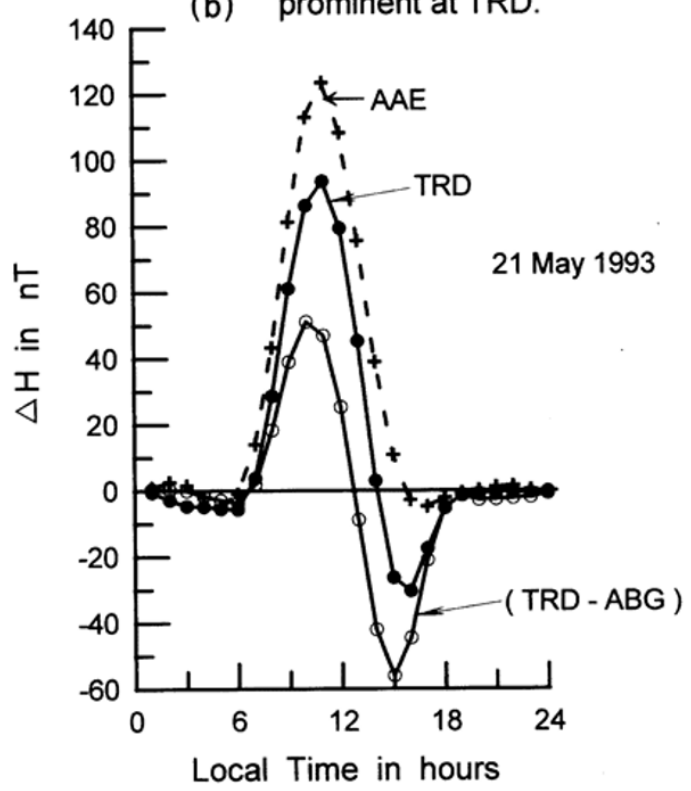

Fig. 9. (a) is a day (3 June 1992) with the prominent occurrence of ECEJ (1500-1800 LT) at AAE. 21 May 1993 is an example of strong ECEJ at TRD (b). The curve of electrojet strength is representative of the occurrence of ECEJ at the Indian longitude.

pared to the reduced amplitude in the variation at TRD. Contrary to this, on 14 June 1993 (ECEJ; 1500-1800 LT), the diurnal variation curve of the ' $H$ ' field at TRD is more pronounced than that at AAE. Completely distorting behaviour in the diurnal curves depending on the time of occurrence of CEJ needs to be dealt in more detail by the extensive analysis of individual events, based on a narrow classification of local time intervals and events.

Two singular cases of the occurrences of ECEJ (15001800 LT) at AAE and TRD are shown in Fig. 9. 3 June 1992 is a case where ECEJ at AAE is prominent, but the noon field at AAE and TRD are of comparable magnitude. 21 May 1993 is a day with a strong negative field present at TRD, but rather insignificant southward field at AAE during 1500-1800 LT. Variable influence on the diurnal field in 


\section{Longitudinal Departures in $\triangle \mathrm{H}(\mathrm{CEJ})$ with Local Time}

$(1100-1200$ LT $)$ NCEJ

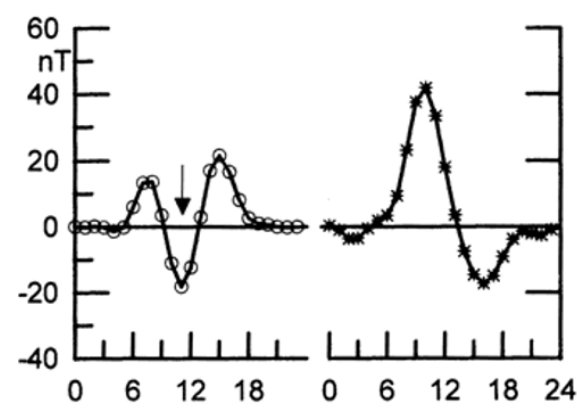

$(1300-1400$ LT) ACEJ

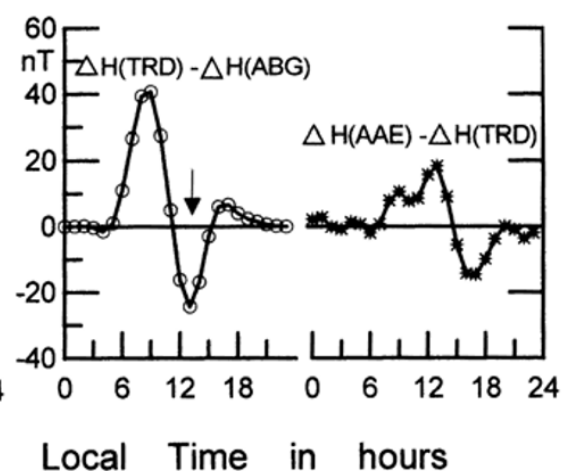

$(1500-1800$ LT) ECEJ

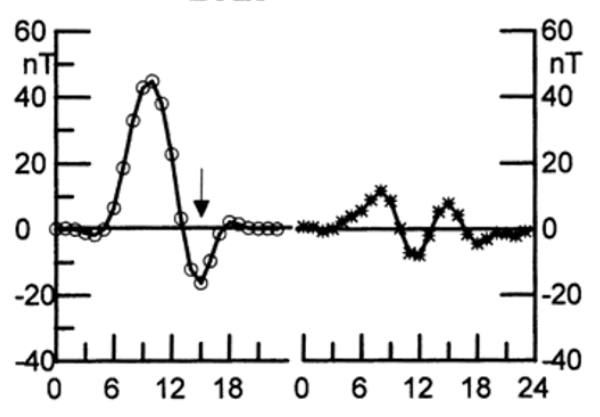

Fig. 10. Systematic modification in the current system effecting the semi-diurnal mode of variation for three local time intervals of CEJ conditions over the two equatorial latitudes, separated in longitude by 2 hours is shown.

total implies the associated contribution from the day-to-day variations in the wind induced dynamo itself.

The progressive influence of the local time characteristics of the equatorial counter electrojet at the two locations TRD and AAE are summarized in Fig. 10. The manifestation of the CEJ influence during pre-noon hours and post-noon hours as represented by $\Delta H(\mathrm{TRD})-\Delta H(\mathrm{ABG})$ and the average difference fields for local times are also shown. The striking feature to note is that in the event of definite NCEJ, the difference field in the pre-noon hours exhibits a peak value of $+40 \mathrm{nT}$ and the afternoon magnitude of the order of $-20 \mathrm{nT}$, suggesting the augmented intensity of the current system over the longitude of AAE at the time when the field at TRD is effected by the strong westward equatorial current system. The phenomenon of negative difference field seen in the afternoon hours is a persistent feature for all the three cases, irrespective of the occurrence time of CEJ. Systematic reduction in the pre-noon field, with the passage of local time of occurrence condition of CEJ, is an evidence of the modification of the entire current system associated with the Sq field and the electrojet.

No events could be traced during (0900-1100 LT), may be due to the large positive gradients in the conductivity and electric field prevailing during this period. Morning and afternoon hours when the CEJ's are most frequent, global scale eastward electric field Ey can be smaller and the wind generated current may become comparable to or greater than that due to Ey even at the magnetic equator (Reddy and Devasia, 1981).

\section{Discussion}

It is well known that the changes in the horizontal component of the Earth's magnetic field is proportional to the product of the conductivity and the east-west electric field. Stening (1977) emphasized that the EEJ current and the Sq current are driven mainly by the electric fields that originate in the global wind dynamo and its associated day-to-day variations resulting in the variability of equatorial electrojet along with that of the Sq current system. The strength of the equatorial electrojet is controlled by the eastward electric field, neutral winds, longitudinal gradients of the electric fields and conductivities. Referring to the results of the longitudinal comparison of the local time dependence features of CEJ and its influence on the day-to-day variability, it is evident from Figs. 1 and 2, that the diurnal patterns at both the longitude locations match well in the morning sector although the afternoon field at AAE which comes under the electrojet influence drops much faster. As the conductivity factor is not expected to change drastically on such periods, the early collapse of the afternoon field at AAE prior to that at TRD, may be attributed to the change in the longitudinal gradient in the primary east-west electric field (Gagnepain et al., 1976). The average feature of the concurrent occurrences in the MCEJ (Fig. 2), observed at both the locations, is almost a regular phenomena as observed by Mayaud (1977).

Most frequent and simultaneous occurrence at TRD and AAE are observed on days of Evening Counter electrojet (ECEJ) events (Fig. 7). The distinct minimum observed in the ' $H$ ' field during this interval (1500-1800 LT) was evident at both the locations for $75 \%$ of occasions, wherein the reference was with respect to the events chosen for known ECEJ occurrence at TRD. This is in conformity with the findings of Marriott et al. (1979), who have suggested that the chance of an ACEJ occurrence at two stations, separated in longitude, on the same day becomes greater if it had occurred in the eastern station earlier. Varied distinct characteristics in the diurnal magnitude are evident from the occurrence time of the CEJ at the Indian longitude. The longitudinal effects on the NCEJ (1100-1200 LT) and ACEJ (13001400 LT) as shown in Figs. 5 and 6 clearly suggest the shifting of the expected noon time maximum at AAE to the early hours coinciding with the maximum at TRD whereas no perceptible noon time dip is observed at AAE. The average 
Table 1.

\begin{tabular}{|c|c|c|c|c|}
\hline S. no. & TYPE & Represented as & Nos. of Cases & EEJ Strength \\
\hline 1. & Strong Electrojet & SEJ & 56 cases & $>70 \mathrm{nT}$ \\
\hline \multirow[t]{2}{*}{2.} & Morning Counter Electrojet & MCEJ (0600 LT) & 20 cases & - \\
\hline & & MCEJ (0700-0800 LT) & Individual cases & \\
\hline 3. & Noon Counter Electrojet & NCEJ (1100-1200 LT) & 6 cases & - \\
\hline 4. & Afternoon Counter Electrojet & ACEJ (1300-1400 LT) & 12 cases & - \\
\hline 5. & Evening Counter Electrojet & ECEJ (1500-1800 LT) & 24 cases & - \\
\hline
\end{tabular}

phenomena observed well infer the modification of the normal eastward field originated from the global Sq in the noon hours at both the locations. However, there exists a distinct magnitude of depression at TRD in the noon hours. The westward field responsible for NCEJ at TRD does not seem to influence the variation at AAE, which lies within two hours apart in longitudinal extent. Rastogi (1973) suggested the possibility that the ACEJ can be fairly localized in nature and may not occur concurrent at two stations even if they are separated only by $2-3$ hours longitude. Kane (1973) reported such cases to be due to short-lived equatorial wind typhoons at ionospheric heights. Rastogi (1974b) showed that the occurrence of the morning and ACEJ events is correlated with the time of maximum effect of the lunar semi-diurnal magnetic variation. Later Forbes and Lindzen (1976), Stening (1977), Marriott et al. (1979) and Hanuise et al. (1983) in their calculation of dynamo electric field over the equator have shown the predominance of the contribution to the equatorial electric field from the semi-diurnal tidal modes $S_{2}$ $(2,2)$ and $S_{2}(2,4)$ in producing the CEJ pattern at $0600 \mathrm{hr}$ and $1800 \mathrm{hr}$ LT. Stening (1995) ascribed the major contribution of the day-to-day variations in EEJ and CEJ to various semi-diurnal tidal modes such as $(2,2),(2,3)$ and $(2,4)$ superposed on the global current system. Model calculations by Marriott et al. (1979) and Hanuise et al. (1983) suggested that ACEJ can well be formed owing to a proper combination of phases and amplitude of the tidal wind modes in the dynamo region. Asymmetric diurnal trend observed in the difference curves presented in Fig. 10, demonstrate the dominance of the semi-diurnal tidal modes of varying magnitude depending on the occurrence time of the CEJ events at the equator. Somayajulu (1988) discussed the changes in the relative amplitudes and phases of various harmonic components associated with CEJ events and suggested the amplification of the semi-diurnal and ter-diurnal components compared to the diurnal components during CEJ days. The random component current systems discussed by MacDougall (1979) mentioned the current systems associated with the day-to-day variability of the electrojet to be caused by localized dynamo region source near the equator. Ananda Rao (1976) and Ananda Rao et al. (1977) investigated the effects of horizontal wind shears and the effects of vertical winds to the observed CEJs. The limited longitudinal extent of the phenomenon of occurrence of CEJ conditions at TRD, during the noon hours could necessarily be attributed to the influence of local winds confined to a narrow longitude zone. The abnormalities demonstrated by the local time character- istics of the CEJ events are in conformity with the theoretical findings of earlier researchers on vertical local winds and neutral wind shears (Raghava Rao and Ananda Rao, 1980; Kane and Trivedi, 1981; Rangarajan and Rastogi, 1993).

From the results presented in Fig. 10, it is ascertained that with the passage of time of occurrence of CEJ conditions progressively from noon (1100-1200 LT and 1300 $1400 \mathrm{LT})$ to evening (1500-1800 LT) hours, the local wind generated distortions in the semi-diurnal trend in the difference field of the electrojet is seen to diminish significantly towards the evening hours effecting reduced scales of difference field during evening CEJ conditions.

\section{Conclusion}

During SEJ, MCEJ and Evening counter electrojet events, the diurnal patterns at TRD and AAE almost correspond.

Most frequent and simultaneous occurrence of CEJ at both the equatorial stations TRD and AAE are found on days of ECEJ. The amplitude of the northward diurnal component decreases considerably, depending on the strength of the semi-diurnal mode active over the location. Common occurrence of the depression could be observed in the evening times especially when the ' $H$ ' field due to the ionospheric currents are already in its low magnitude.

Limited longitudinal extent of the CEJ phenomenon observed during NCEJ (1100-1200 LT) and ACEJ (1300-1400 LT) events at Trivandrum is attributed to the local wind generated distortions in the equatorial electrojet. As the counter electrojet advances from noon to the evening hours, the magnitude of the longitudinal asymmetry in the ' $H$ ' component seem to alter considerably, wherein the variability of the difference field also exhibit negligible departures with local time. Thus the results presented clearly emphasize the role of the local wind shears modifying the jet fields especially during the noon hours in addition to the changes in the large scale global wind dynamo supporting the suggestions by Reddy and Devasia (1981).

\section{References}

Ananda Rao, B. G., Effects of gravity wave winds and wind shears on equatorial electrojet, Geophys. Res. Lett., 3, 545-547, 1976.

Ananda Rao, B. G., R. Ragahava Rao, and C. Raghava Reddi, Electric fields by gravity wave winds in the equatorial electrojet, J. Geophys. Res., 82, $1513,1977$.

Bartels, J. and H. F. Johnston, Geomagnetic tides in horizontal intensity at Huancayo, Terr. Mag. Atmos. Elec., 45, 269-308, 1940.

Chapman, S., The equatorial electrojet as detected from the abnormal electric current distributions above Huancayo, Peru and elsewhere, Arch. Meteorol. Geophys. Bioclimatol., A4, 368-390, 1951. 
Egedal, J., The magnetic diurnal variation of the horizontal force near the magnetic equator, J. Geophys. Res., 52, 449, 1947.

Egedal, J., Daily variation of the horizontal magnetic force at the magnetic equator, Nature, 161, 443-449, 1948.

Forbes, J. M. and R. S. Lindzen, Atmospheric solar tides and their electrodynamic effects: II-The equatorial electrojet, J. Atmos. Terr. Phys., 38, 911-920, 1976.

Gagnepain, J., M. Crochet, and A. D. Richmond, Theory of longitudinal gradients in the equatorial electrojet, J. Atmos. Terr. Phys., 38, 279-286, 1976.

Gouin, P., Reversal of the magnetic daily variation at Addis Ababa, Nature, 193, 1145-1146, 1962

Gouin, P. and P. N. Mayaud, A propo dell existence possible dun "Counter Electrojet" aux latitudes magnetiques equatorials, Ann. Geophys., 23, 41-47, 1967.

Hanuise, C., C. Mazaudier, P. Vila, M. Blanc, and M. Crochet, Global dynamo simulation of ionospheric currents and their connection with the equatorial electrojet and counter electrojet-A case study, J. Geophys. Res., 88, 253-270, 1983.

Hutton, R. and J. O. Oyinloye, The Counter Electrojet in Nigeria, Ann. Geophys., 26, 921-926, 1970.

Kane, R. P., Longitudinal extent of the Counter Electrojet, Physical Research Laboratory, Report AER 73-08, Ahmedabad, 1973.

Kane, R. P. and N. B. Trivedi, Confinement of Equatorial Counter Electrojet to restricted longitudes, J. Geomag. Geoelectr., 33, 379-382, 1981.

MacDougall, J. W., Equatorial Electrojet and Sq Current System Part I, J. Geomag. Geoelectr., 31, 341-358, 1979.

Marriott, R. T., A. D. Richmond, and S. V. Venkateswaran, The Quiet-time Equatorial Electrojet and Counter Electrojet, J. Geomag. Geoelectr., 31, 311-340, 1979.

Mayaud, P. N., The Equatorial Counter Electrojet. A review of its geomagnetic aspects, J. Atmos. Terr. Phys., 39, 1055-1070, 1977.

Onwumechilli, A., Lunar effects on the diurnal variation of the geomagnetic horizontal field near the magnetic equator, J. Atmos. Terr. Phys.,
25, 55-70, 1963.

Onwumechilli, A. and S. I. Akasofu, On the abnormal depression of $\mathrm{Sq}(\mathrm{H})$ under the equatorial electrojet in the afternoon, J. Geomag. Geoelectr., 24, 161-173, 1972.

Raghava Rao, R. and B. G. Ananda Rao, Vertical winds as a plausible cause for equatorial electrojet, Geophys. Res. Lett., 7, 357-360, 1980.

Rangarajan, G. K. and R. G. Rastogi, Longitudinal difference in magnetic field variations associated with quiet day counter electrojet, J. Geomag. Geoelectr., 45, 649-656, 1993.

Rao, K. N. and K. S. Rajarao, Quiet day magnetic variations near the magnetic equator, Nature, 200, 460-461, 1963.

Rastogi, R. G., Counter equatorial electrojet currents in the Indian zone, Planet. Space Sci., 21, 1355-1365, 1973.

Rastogi, R. G., Westward Equatorial Electrojet during daytime hours, $J$. Geophys. Res., 79, 1503-1512, 1974a.

Rastogi, R. G., Lunar effects in the counter electrojet near the magnetic equator, J. Atmos. Terr. Phys., 36(1), 167-000, 1974b.

Reddy, C. A. and C. V. Devasia, Height and latitude structure of electric fields and currents due to local East-West winds in the equatorial electrojet, J. Geophys. Res., 86, 5751-5767, 1981.

Sastry, N. S. and R. W. Jayakar, Afternoon depression in horizontal component of the geomagnetic field at Trivandrum, Ann. Geophys., 28(3), 589-591, 1972.

Somayajulu, V. V., Behaviour of harmonic components of the geomagnetic field during counter electrojet, J. Geomag. Geoelectr., 40, 111-130, 1988.

Stening, R. J., Analysis of contributions to Ionospheric dynamo currents from e.m.f.'s at different latitudes, Planet. Space Sci., 25, 587-594, 1977.

Stening, R. J., What drives the equatorial electrojet?, J. Atmos. Terr. Phys., 57, 1117-1128, 1995.

S. Alex (e-mail: salex@iig.iigm.res.in) and S. Mukherjee 